\title{
Biological activity of the thyroid TRK-T3 oncogene requires signalling through Shc
}

\author{
E Roccato', C Miranda', V Ranzi', M Gishizki', MA Pierotti' and A Greco*,I \\ 'Department of Experimental Oncology, Istituto Nazionale Tumori, Via G. Venezian I, 20133 Milan, Italy; ${ }^{2}$ SUGEN, 230 East Grand Ave, South San Francisco, \\ California, CA 94080, USA
}

The thyroid TRK-T3 oncogene, produced by a chromosomal translocation, is a chimeric, constitutively activated version of the NTRKI/NGF receptor and it is able to transform NIH3T3 cells and differentiate PCI2 cells. TRK-T3 oncoprotein triggers multiple signal transduction pathways. Among others, TRK-T3 binds and phosphorylates the Shc and SNTI/FRS2 adaptor proteins both involved in coupling the receptor tyrosine kinase to the mitogen-activated protein kinase pathway by recruiting Grb2/SOS. We were interested in defining the role of Shc in the oncogenesis by TRK-T3. The mutation of TRK-T3 tyrosine 291, docking site for both Shc and FRS2, abrogates the oncogene biological activity. To directly explore the role of Shc we used the ShcY3I7F mutant, which carries the mutation of a tyrosine residue involved in Grb2 recruitment. We demonstrated that the ShCY3I7F mutant exerts an inhibitory effect on TRK-T3 transforming activity. Such effect can be modulated by the amount of ShcY3 I7F protein and affects the viability of cells expressing TRK-T3 by means of a mechanism involving apoptosis. Our results indicate a definitive role of the adaptor protein Shc in TRK-T3 transforming activity.

British Journal of Cancer (2002) 87, 645 -653. doi: I 0.1038/sj.bjc.6600544 www.bjcancer.com

(c) 2002 Cancer Research UK

Keywords: TRK-T3; Shc; NTRKI; signal transduction; transforming activity

The thyroid TRK oncogenes are created by chromosomal rearrangements fusing the tyrosine kinase (TK) domain of the NTRK1/ NGF receptor to the $5^{\prime}$-end portion of different activating genes (Pierotti et al, 1996). In the TRK-T3 oncogene, produced by a $\mathrm{t}(1 ; 3)$ translocation, the NTRK1 TK domain is juxtaposed to sequences of the TFG gene (Greco et al, 1995), which encodes a protein of unknown function containing a coiled-coil domain, putative phosphorylation sites for PKC and CK2 and glycosylation sites (Mencinger et al, 1997; Mencinger and Aman, 1999). The TFG coiled-coil domain plays an important role in TRK-T3 activation and its consequent biological activity insofar as it mediates the dimerization leading to the ligand-independent tyrosine phosphorylation of the TK domain. Moreover, also the regions outside the coiled-coil domain are required for TRK-T3 transforming activity (Greco et al, 1998) in ways that are currently under investigation.

Signalling by the NTRK1 receptor has been extensively studied and it involves both Ras-dependent and Ras-independent pathways. Ligand activation leads to the phosphorylation of five tyrosine residues in the intracellular domain of NTRK1: Tyr490 in the juxtamembrane region, Tyr670, Tyr674, Tyr675 within the TK domain, and Tyr785 in the carboxyl-terminal tail. Phosphorylated Tyr490 provides the docking site for and mediate the phosphorylation and activation of Shc and SNT1/FRS2, two adaptor proteins signalling through the Ras/MAPK cascade; phosphorylated Y785 recruits and activates PLC $\gamma$ (Kaplan and Miller, 1997; Meakin et al, 1999). The other three tyrosines are required for kinase activity, and recent studies have shown that they are also involved in the activation of rAPS and SH2-B (Qian et al, 1998) and the direct recruitment of Grb2 (MacDonald et al,

*Correspondence: A Greco; E-mail: greco@istitutotumori.mi.it Received 19 February 2002; revised 25 June 2002; accepted 26 June 2002
2000). The common downstream effectors of Shc, FRS2, Grb2, rAPS and SH-2B are ERK1/2 MAP kinases, followed by the activation of transcription factors and the induction of immediate early genes.

Shc adaptor proteins are cytoplasmic substrates for a number of receptor and non-receptor tyrosine kinases (TKs), including NTRK1 (van der Geer et al, 1995). By both alternative splicing and transcription start sites three protein isoforms of 66,52 and $46 \mathrm{kDa}$ are produced. The Shc p52/46 isoforms are involved in the transmission of signals from activated TKs (Migliaccio et al, 1997), and the Shc p66 isoform controls stress apoptotic responses and life span (Migliaccio et al, 1999). In addition to collagen homology $(\mathrm{CH})$ domains, Shc proteins have phosphotyrosine binding (PTB) and Src homology 2 (SH2) domains (Migliaccio et al, 1997; Pelicci et al, 1992) both involved in exclusive or cooperative binding to phosphotyrosine residues. Upon receptor binding, Shc undergoes phosphorylation at three tyrosine residues $(317,239$ and 240), becomes capable of interacting with the $\mathrm{SH} 2$ domain of Grb2, and causes the activation of the SOS/Ras/MAPK pathway. Different roles have been ascribed to Tyr317 and Tyr239/240, depending on the cell environment and on the kinase that trigger Shc activation. In hematopoietic cells and in EGF-stimulated NIH3T3 phosphorylation of Tyr317 has been proposed to trigger MAPK activation. On the contrary, phosphorylated Tyr239/240 signal to c-myc and thus regulate IL-3 dependent apoptosis in haematopoietic cells and contribute to the EGF-induced proliferation of NIH3T3 fibroblasts (Gotoh et al, 1996, 1997). In RAT-2 cells transformed by VEGF receptor, Tyr239/240 mediate an inhibitory signal for cell growth (Fournier et al, 1999). In PC12 cells Tyr239/240, but not Tyr317, play an important contribution to the NGF-induced Ras/MAP kinase activation and differentiation (Thomas and Bradshaw, 1997). It is therefore possible that the signalling pathway of Tyr239/240 may involve effectors other than Grb2. 
The involvement of Shc in NTRK1 signal transduction has been demonstrated by various studies (Obermeier et al, 1993; Stephens et al, 1994). In a previous work we detected interaction of Shc SH2 domain with the wild type NTRK1 receptor and various TRK oncogenes expressed in NIH3T3 cells (Borrello et al, 1994). After the subsequent discovery of the Shc PTB domain, experiments with PC12 cells showed that Shc PTB domain interacts with Tyr490 of the activated NTRK1 receptor, whereas the interaction with an unidentified p115 phosphoprotein was proposed for the SH2 domain (Dikic et al, 1995).

As we are interested in elucidating the signal transduction pathways leading to the TRK oncogene transformation of NIH3T3 cells, we began by investigating the involvement of the proteins known to transduce NTRK1 signals. This apparently obvious approach is not as trivial as it may seem because NGF-induced NTRK1 activation leads to cell type specific biological effects, such as the differentiation of neuronal cells, the apoptosis of medulloblastoma cells and the mitogenesis of non-neuronal cells (Muragaki et al, 1997). This suggests that the critical elements of NTRK1 signalling may vary in cell lines from different tissues. In addition to differences due to cell specificity, TRK oncogenes may trigger signal transduction pathways other than the wild type receptor as a consequence of the properties contributed by structural rearrangements (cytoplasmic localization, the presence of activating sequences).

In a previous study we demonstrated that Shc is activated by TRK oncoproteins and therefore capable to recruit Grb2 (Borrello et al, 1994). We have more recently also determined the activation of FRS2, PLC $\gamma$, ERK1/2 and JNK MAP kinases in cells transformed by TRK-T3 (Greco et al, manuscript in preparation), as well as the recruitment of IRS1 and IRS2 by NTRK1 and TRK-T1 oncogene (Miranda et al, 2001).

In the study reported here we investigated the contribution of the Shc adaptor in TRK-T3 signal transduction. The mutation of the docking site for both Shc and FRS2 abolished the TRK-T3 biological activity, thus indicating that possible other pathways might not overcome the lack of the two adaptors. To further define the role of Shc in TRK-T3 activity we used the ShcY317F dominant-negative mutant, in which one of the tyrosines recruiting the Grb2/SOS complex has been mutated to phenylalanine. Our data show that ShcY317F reduces TRK-T3 biological activity, thus indicating the indispensability of Shc. Moreover, we report evidences that cells expressing both TRK-T3 and ShcY317F undergo cell death by a mechanism involving apoptosis.

\section{MATERIALS AND METHODS}

\section{Plasmid construction}

The T3/WT plasmid contains the TRK-T3 cDNA inserted into the pRC/CMV expression vector (Greco et al, 1995), which carries the neomycin resistance gene. The T3/Y291F mutant carries the mutation of Tyr291 to phenylalanine (F291) and was constructed by means of site-directed mutagenesis using an in vitro oligonucleotide mutagenesis system (Altered Sites in vitro Mutagenesis System, Promega). The T3/ABN kinase-defective mutant carries the mutation of the ATP binding site Lys339 to Ala (Greco et al, 1998). In ShcY317F mutant the Tyr317 is mutated to Phe. The cDNAs encoding ShcWT and ShcY317F are inserted in the pCGN expression vector, containing the hygromycin-resistance gene, and are tagged by the HA epitope at the $\mathrm{N}$-terminus.

The VGF8-luc is a reporter plasmid made of $v g f$ promoter sequence fused to the luciferase reporter gene (a kind gift from Dr R Possenti).

The pRL-CMV vector, containing the coding region of the Renilla luciferase (RL) gene, was provided by Promega.

\section{Cell culture and transfection}

Mouse NIH3T3 fibroblasts were cultured in Dulbecco's modified Eagle's medium (DMEM) supplemented with $10 \%$ calf serum, human kidney $293 \mathrm{~T}$ cells in DMEM supplemented with $10 \%$ foetal calf serum, and transformed cell lines in DMEM supplemented with 5\% calf serum. PC12 cells were grown in RPMI-1640 medium supplementd with $5 \%$ foetal calf serum and $10 \%$ horse serum. NF797 cells are NIH3T3 cells transformed by the TRK-T3 oncogene; NWT and NY317F cell lines are derived from NIH3T3 cells respectively transfected with ShcWT and ShcY317F plasmids, and selected in the presence of hygromycin $\left(25 \mu \mathrm{g} \mathrm{ml}^{-1}\right)$. 3.9HG are semi-transformed cells generated by the transfection of NY317F cells with the TRK-T3 oncogene and isolated in medium supplemented with $5 \%$ calf serum plus hygromycin $\left(25 \mu \mathrm{g} \mathrm{ml}^{-1}\right)$ and G418 $\left(400 \mu \mathrm{g} \mathrm{ml}^{-1}\right)$.

The NIH3T3, NWT and NY317F cells $\left(8 \times 10^{4} / 60 \mathrm{~mm}\right.$ plate $)$ were transfected by the $\mathrm{CaPO}_{4}$ method as previously described (Bongarzone et al, 1989), using $250 \mathrm{ng}$ of expression plasmid DNA together with $15 \mu \mathrm{g}$ of mouse DNA. Transformed foci were selected in DMEM supplemented with 5\% serum, in the presence or absence of hygromycin $\left(25 \mu \mathrm{g} \mathrm{ml}^{-1}\right), \mathrm{G} 418\left(400 \mu \mathrm{g} \mathrm{ml}^{-1}\right)$ or both. G418- and hygromycin-resistant colonies were selected in DMEM plus $10 \%$ serum containing the selective agents at the above concentrations. The transformed foci and resistant colonies were either fixed or isolated for further studies 2 weeks after transfection. The 293T cells were transiently cotransfected with T3/WT or T3/mutants $(1 \mu \mathrm{g})$ together with Shc constructs or pCGN empty vector $(5 \mu \mathrm{g})$ using the $\mathrm{CaPO}_{4}$ method. The cells were kept in $10 \%$ serum medium for $6-7 \mathrm{~h}$, serum-starved overnight in DMEM containing $0.5 \% \mathrm{FCS}$, and then processed for protein extraction. PC12 cells were transfected using Cellfectin (Lifetechnologies, Inc.). Cells $\left(2 \times 10^{5}\right)$ were seeded on collagen-coated 12-multiwell plates and transfected with $400 \mathrm{ng}$ of T3/WT or T3/Y291F plasmid. In cotransfection experiments $100 \mathrm{ng}$ of $\mathrm{T} 3 / \mathrm{WT}$ together with $500 \mathrm{ng}$ of ShcWT, ShcY317F or pCGN plasmid were used. Specific plasmid DNA were transfected together with $150 \mathrm{ng}$ of VGF8-luc plasmid and $50 \mathrm{ng}$ of pRL-CMV plasmid (Promega). Cells were incubated with the reagent for $7 \mathrm{~h}$ and scored for neurites outgrowth 2 days later.

\section{Luciferase activity assay}

Both luciferase activities of VGF8-luc and pRL-CMV genes in PC12 cells lysates were measured using the Dual-luciferase reporter assay system (Promega). Assays were performed according to the manufacturer's recommendations. Light emission was measured using a TD-20/20 Luminometer.

\section{Microfocus forming assay}

The assay was performed as described by Li et al (1999). One hundred cells from NF797 cell lines transfected with pCGN vector, ShcWT or ShcY317F were combined with $1 \times 10^{5}$ NIH3T3 cells in $10-\mathrm{cm}$ dishes, and cultured for 2 weeks in medium containing $5 \%$ calf serum. The foci were counted after GIEMSA staining.

\section{Immunoprecipitation, pull-down and Western blot analysis}

Cells were lysed with PLCLB buffer (50 mM HEPES, $150 \mathrm{~mm} \mathrm{NaCl}$, $10 \%$ glycerol, $1 \%$ Triton X-100, $1.5 \mathrm{~mm} \mathrm{MgCl}_{2}, 1 \mathrm{mM}$ EGTA, $10 \mathrm{mM} \mathrm{Na} \mathrm{P}_{2} \mathrm{O}_{7}, 100 \mathrm{mM} \mathrm{NaF}$ ) supplemented with aprotinin, pepstatin, leupeptin, PMSF and $\mathrm{Na}_{3} \mathrm{VO}_{4}$. One milligram of the cell extracts was precipitated with the appropriated antibodies, or with GST-Grb2(SH2) fusion protein conjugated to gluthatione-sepharose, or with p13sucl-agarose. The precipitates were washed three 
times with HNTG buffer (20 mM HEPES, $150 \mathrm{~mm} \mathrm{NaCl}, 0.1 \%$ Triton X-100, 10\% glycerol) and boiled in Laemmli sample buffer. Protein samples were electrophoresed on $8.5 \%$ SDS-PAGE, transferred onto nitrocellulose filters and immunoblotted with the appropriated antibodies. The immunoreactive bands were visualised using horseradish peroxidase-conjugated secondary antibody and enhanced chemiluminescence (Amersham). The anti-TRK and anti-Grb2 antibodies were from Santa Cruz Biothec., Inc.; the anti-HA antibodies were from BabCo; the anti-Shc, antiFRS2, anti-phosphotyrosine antibodies and the p13sucl-agarose were from Upstate biothecnology, Inc.

\section{TUNEL analysis of DNA fragmentation}

In situ detection of apoptotic cells was performed on adherent cells cultured on chamber slides by using the In Situ Cell Death Detection Kit, Fluorescein (Boehringer Mannheim), according to the manufacturer's instruction. The slides were then counterstained with 4',6-diamin2-phenylindole.

\section{RESULTS}

\section{TRK-T3 biological activity requires the Shc/FRS2 docking site}

The Tyr490 residue of the NTRK1 receptor is involved in the recruitment and activation of Shc adaptor proteins; such interaction occurs through the Shc PTB domain (Dikic et al, 1995). Tyr490 is also the docking site for SNT1/FRS2, a myristilated adaptor molecule signalling through Grb2/SOS/ERKs (Rabin et al, 1993; Kouhara et al, 1997; Ong et al, 2000). In vitro studies have shown a competition between Shc and FRS2 for the binding to the phosphorylated Tyr490 of NTRK1 (Meakin et al, 1999). With the final aim to provide evidences of possible oncogene-specific signal transduction pathways that could bypass the need for Shc and FRS2, we defined the role of TRK-T3 Tyr291 (corresponding to NTRK1 Tyr490) in the oncogene biological activity. The tyrosine residue was changed to phenylalanine by site-directed mutagenesis, to produce T3/Y291F (Figure 1). Wild type and Y291F TRK-T3 cDNAs were inserted into the pRC/CMV mammalian expression vector, which also carries the neomycin resistance gene. Transient expression into human kidney $293 \mathrm{~T}$ cells showed that, similarly to the wild type, the $\mathrm{T} 3 / \mathrm{Y} 291 \mathrm{~F}$ mutant produced a phosphorylated $68 \mathrm{kDa}$ protein (Figure 2A). Western blot analysis of Shc immunocomplexes hybridised with anti-TRK (Figure 2A) showed that the $\mathrm{T} 3 / \mathrm{Y} 291 \mathrm{~F}$ mutant is unable to interact with Shc (Figure 2A). The same cell extracts were incubated with the FRS2 interacting protein p13sucl conjugated to agarose beads. The complexes immunoblotting with anti-FRS2 antibodies showed a constitutive association of FRS2 with p13suc1. However, immunoblotting with anti-phosphotyrosine antibodies showed that FRS2 is activated by TRK-T3 wild type but not by the T3/Y291F mutant.

We next analysed the transforming activity of T3/Y291F mutant by NIH3T3 transfection/focus formation experiments. As reported in Figure 2B, the transfection efficiency of T3/Y291F, determined as frequency of G418-resistant colonies, was slightly higher than wild type. However, the capacity to induce NIH3T3 transformation was completely abolished in the mutant oncogene. The biological effect of T3/Y291F mutant was also investigated in PC12 cells, in which the constitutively activated TRK oncogenes recapitulate the neuronal differentiating effect of NGF-stimulated NTRK1 receptor. As shown in Figure $2 \mathrm{C}$ T3/Y291F failed to induce neurites outgrowth. To quantify the differentiating activity of the TRK-T3 constructs we performed PC12 transfection in the presence of the VGF8-luc reporter plasmid; the latter is made of the luciferase reporter gene driven by the promoter of the $v g f$, a gene induced by NGF and considered a differentiation marker (Possenti et al, 1992; Canu et
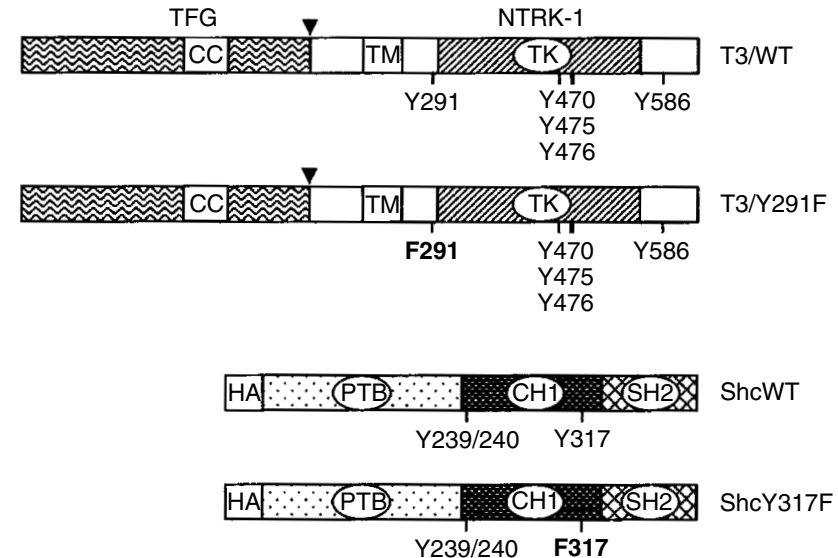

Figure I Schematic representation of TRK-T3 and Shc constructs. In the TRK-T3 constructs the portions contributed by TFG and NTRKI are shown, with an arrowhead indicating the breakpoint. The coiled-coil (CC), transmembrane (TM) and tyrosine kinase (TK) domains are indicated. The tyrosine residues involved in Shc and FRS2 interaction (Y29I), PLC $\gamma$ interaction (Y586) and tyrosines of the activation loop (Y470, Y475 and Y476) are indicated. In T3/Y29IF mutant the tyrosine 29I has been mutated to phenylalanine (F29I). The TRK-T3 cDNAs were inserted into the pRC/CMV expression vector. The Shc constructs show the PTB domain, the collagen homology region $(\mathrm{CHI})$ and the $\mathrm{SH} 2$ domain. Y239/240 and Y317 are tyrosine residues phosphorylated by tyrosine kinases. In ShcY3I7F, tyrosine 317 is mutated to phenylalanine (F3/7). The Shc cDNAs contain the HA epitope at the $\mathrm{N}$-terminus and were inserted into the pCGN mammalian expression vector.

$a l, 1997)$. As shown by the graph in Figure 2C, the luciferase activity in cells expressing $\mathrm{T} 3 / \mathrm{Y} 291 \mathrm{~F}$ is drastically reduced $(86 \%)$ with respect to $\mathrm{T} 3 / \mathrm{WT}$.

All these results demonstrated that the Y291F mutation abrogates the TRK-T3 interaction with Shc and FRS2 adaptors and, consequently, its biological activity. This demonstrates that possible novel signal transduction pathways triggered by the rearranged versions of the NTRK1 receptor may not compensate the need of Shc and FRS2 signalling.

\section{Effect of ShcY317F mutant on TRK-T3-dependent signal transduction}

As a tool for defining the role of Shc adaptor in TRK-T3 signal transduction we used the ShcY317F mutant of the $52 \mathrm{kDa}$ Shc isoform (Figure 1), in which the tyrosine 317 has been changed to phenylalanine. Tyr317 of Shc, in addition to Tyr239/240, recruits Grb2 upon phosphorylation; the ShcY317F mutant has been shown to exert a dominant-negative effect in different biological processes mediated by Shc ( $\mathrm{Li}$ et al, 1999; Stevenson et al, 1999; Boney et al, 2000; Mercalli et al, 2001). The ShcY317F and the control ShcWT constructs (Figure 1) were tagged by the haemoagglutinin (HA) epitope, which confers a small electrophoretic shift with respect to the endogenous p52 Shc, and cloned into the pCGN expression vector harbouring the resistance to hygromycin.

We investigated the capability of ShcY317F to interact with TRK-T3 oncoprotein. Human kidney 293T cells were transiently cotransfected with TRK-T3 or T3/ABN kinase-dead mutant, together with HA-tagged ShcWT, ShcY317F or the pCGN vector as control (Figure 3). The expression of the transfected proteins is shown in Figure 3C. Similarly to ShcWT, the $53 \mathrm{kDa}$ phosphorylated ShcY317F protein coprecipitated with TRK-T3, as shown by the anti-phosphotyrosine Western blot hybridisation of anti-TRK immunocomplexes. This interaction required the phosphorylation 
A

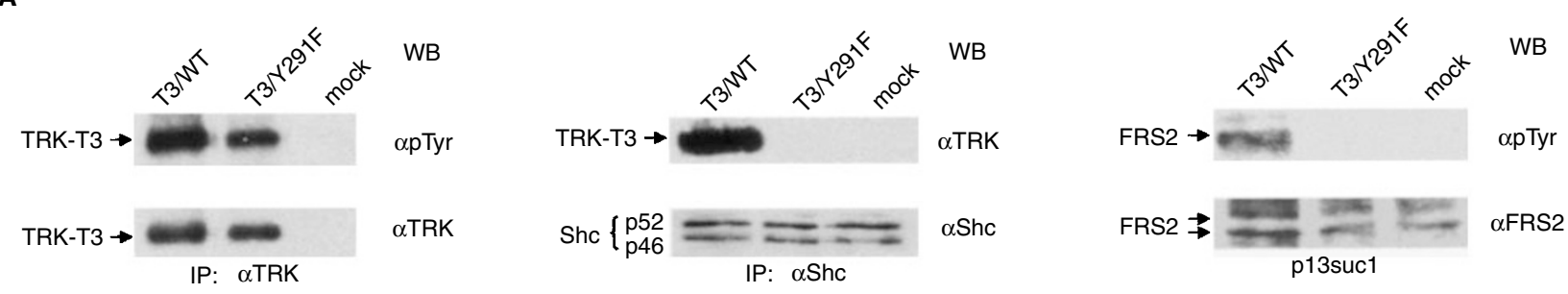

B

\begin{tabular}{|c|c|c|}
\hline Plasmid & G4418 ${ }^{R}$ colonies/ $\mu g D N A$ & Focl/ $\mu g D N A$ \\
\hline Тз/WT & $6.25 \times 10^{3}$ & $2.2 \times 10^{2}$ \\
\hline T3/Y291F & $10.7 \times 10^{3}$ & $<0.01 \times 10^{2}$ \\
\hline Mock & - & $<0.01 \times 10^{2}$ \\
\hline
\end{tabular}

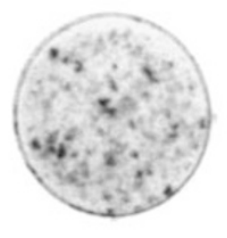

T3/WT

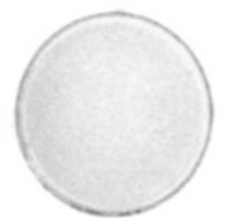

T3/Y291F

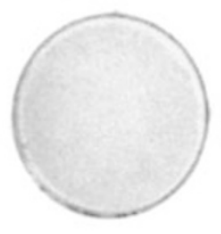

mock
C

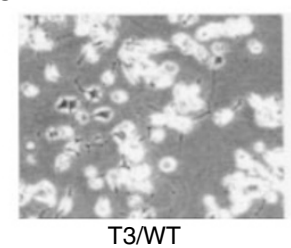

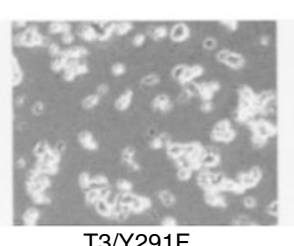

T3/Y291F

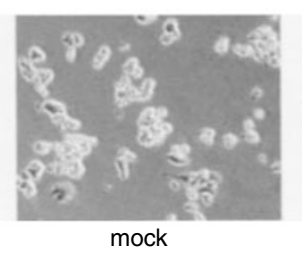

mock

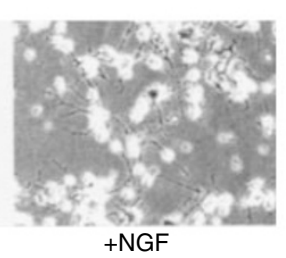

Figure 2 Biochemical and biological analysis of T3/Y29IF mutant. (A) T3/WT or T3/Y29IF expression plasmids were transiently transfected into 293T cells. Cell extracts were immunoprecipitated (IP) with the antibodies anti-TRK, anti-Shc or with pl3sucl-agarose and blotted with anti-phosphotyrosine, anti-TRK, anti-Shc and anti-FRS2 antibodies. (B) Effect of the Y29IF mutation on TRK-T3 transforming activity. NIH3T3 cells were transfected with the indicated constructs and subjected to G4I 8 and foci selection. Plates were fixed and scored after GIEMSA staining 2 weeks later. (C) Effect of the Y29IF mutation on TRK-T3 differentiating activity. PCI2 cells were transfected with the indicated constructs and scored for the presence of neurites 3 days later. As control, untreated and NGF-stimulated $\left(50 \mathrm{ng} \mathrm{ml}^{-1}\right) \mathrm{PCI} 2$ cells are shown. The graph shows a quantification of differentiating activity. TRK-T3 constructs were cotransfected with VGF8-luc and Renilla plasmids; both luciferase activities were measured using the Dual-Luciferase reported assay system (Promega). The values were expressed relative to Renilla luciferase activity for normalization. The results presented are an average of three experiments.

of TRK-T3, as it was undetectable when the T3/ABN kinase-dead mutant (Greco et al, 1998) was used (Figure 3A).

We next investigated the ability of ShcY317F to recruit Grb2 upon TRK-T3 activation. The same cell extracts as above were incubated with the GST-Grb2(SH2) fusion protein, containing the Grb2 SH2 domain. Hybridisation with anti-HA antibodies (Figure 3B, top) showed that the amount of ShcY317F protein reacting with GST-Grb2(SH2) is lower than ShcWT, in accordance with the lack of an interaction site. The ShcY317F-Grb2 interaction was also investigated by coimmunoprecipitation experiments. The above cell extracts were immunoprecipitated with anti-Grb2 antibodies and hybridised with antiphosphotyrosine antibodies. Similarly to ShcWT and endogenous Shc proteins (data not shown), phosphorylated ShcY317F coprecipitated with Grb2 (Figure 3B, bottom). The ShcY317F-Grb2 interaction depends on the TRK-T3-triggered Shc activation, being undetectable when the T3/ABN kinase-dead mutant was used. Grb2 immunocomplexes also contain the phosphorylated TRKT3 oncoprotein (Figure 3B, bottom), as consequence of both direct (MacDonald et al, 2000) and Shc/FRS2-mediated interaction. Altogether these results indicate that ShcY317F is phosphorylated by TRK-T3 at tyrosine residues 239/240 and therefore capable of recruiting Grb2.
As reported in the previous paragraph, both Shc and FRS2 bind, in a competitive manner, to phosphorylated NTRK1 Tyr490 residue (Meakin et al, 1999). We investigated the effect of ShcY317F on FRS2 activation induced by TRK-T3. The same cell extracts from serum-starved 293T cells transiently cotransfected with TRK-T3 and ShcWT, ShcY317F or the pCGN vector as control were pulled-down with the FRS2-interacting p13suc1 protein conjugated to agarose beads, and the eluted complexes analysed by Western blot. As shown in Figure 3D, hybridisation with anti-phosphotyrosine antibodies detected comparable levels of FRS2 phosphorylation in cells expressing TRK-T3 in combination with the pCGN empty vector, ShcWT or the ShcY317F mutant. As control, the expression of FRS2 is shown. These results indicate that, in our experimental conditions, although in the presence of exogenous Shc proteins, Tyr291 of TRK-T3 is still capable to signal through FRS2.

\section{Inhibitory effect of ShcY317F mutant on TRK-T3 biological activity}

In the first paragraph we have shown that the biological activity of TRK-T3 is abrogated by the mutation of the Tyr291, docking site for Shc and FRS2. To directly explore the role of the Shc adaptor in 
A

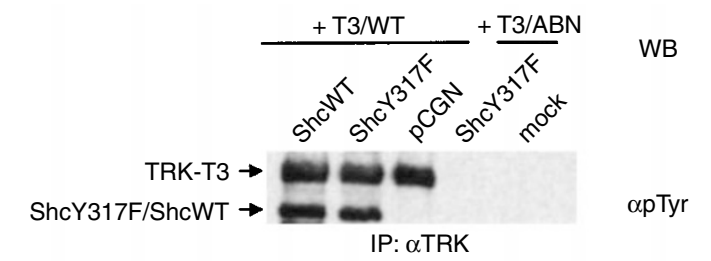

B

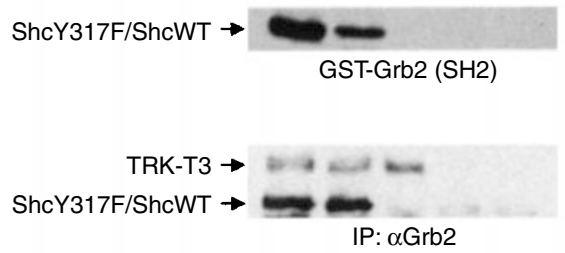

$\alpha \mathrm{HA}$

$\alpha p$ Tyr

C

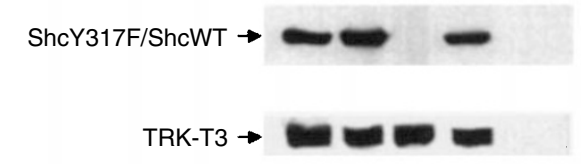

$\alpha H A$

$\alpha$ TRK

D

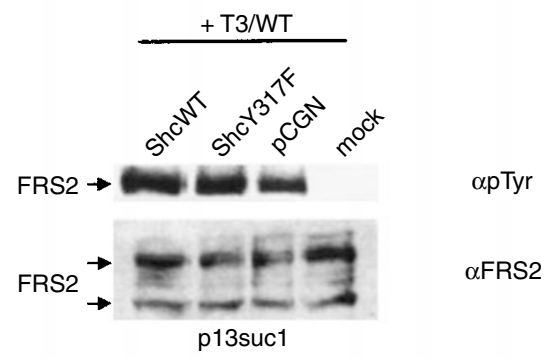

Figure 3 (A) ShcY3 I7F is phosphorylated by TRK-T3. 293T cells were transiently cotransfected with the indicated constructs. Cell extracts were immunoprecipitated (IP) with anti-TRK and immunoblotted (WB) with anti-phosphotyrosine antibodies. The TRK-T3 oncoprotein, the $53 \mathrm{kDa}$ HA-tagged ShcWT and ShcY3I7F transfected proteins are indicated. Cells cotransfected with T3/WT and pCGN vector were used as negative control. (B) Interaction of transfected Shc proteins with Grb2. The above described cell extracts were incubated with the GST-Grb2 $\left(\mathrm{SH}_{2}\right)$ fusion protein (top) or immunoprecipitated with anti-Grb2 antibodies (bottom) and hybridised with anti-HA and anti-phosphotyrosine antibodies, respectively. The phosphorylated proteins present in the anti-Grb2 immunocomplexes are indicated. (C) To show the expression of the transfected proteins, cell extracts above described were immunoblotted with antiHA and anti-TRK antibodies. (D) Effect of ShcY3I7F mutant on TRK-T3 induced FRS2 activation. The same cell extracts above described, except T3/ABN-ShcY3I7F cotransfection, were pulled down with p/3sucl-agarose and immunoblotted with anti-phosphotyrosine or anti-FRS2 antibodies.

TRK-T3 signal transduction, we investigated the effect of the ShcY317F dominant-negative mutant on TRK-T3 biological activity.

We first investigated the effect of ShcY317F on TRK-T3 differentiating activity. PC12 cells were cotransfected with TRK-T3 together with HA-tagged ShcWT, ShcY317F or the pCGN empty vector. The results are reported in Figure 4A. Three days after transfection cell transfected with TRK-T3 and ShcWT or pCGN displayed long neurites. On the contrary, cells transfected with TRK-T3 and ShcY317F showed shorter extentions, with a few of them exceeding the cell diameter. As control the expression of TRK-T3 and HA-tagged Shc proteins is reported (Figure 4A). The differentiating activity was measured by cotransfecting the VGF8-luc reported plasmid, as above described. Luciferase activity in cells expressing T3/WT and ShcY317F was reduced to $50 \%$ with respect to cells expressing T3/WT and ShcWT (graph in Figure $4 \mathrm{~A})$.

We next investigated the effect of ShcY317F mutant on TRK-T3 transforming activity. As first approach we performed the microfocus formation assay. NF797 cells (NIH3T3 transformed by the TRK-T3 oncogene) were transfected with ShcY317F and ShcWT or the empty pCGN vector as control, as described in Materials and Methods. Transfected cells were mixed with an excess of wild type NIH3T3 cells, seeded in medium containing 5\% calf serum, and their ability to form foci was assessed. As shown in Figure $4 \mathrm{~B}$ focus forming capability was unaffected in the NF797 cells transfected with pCGN and ShcWT, but was reduced by about $50 \%$ in the cells expressing ShcY317F.

To more directly explore the effect of ShcY317F on TRK-T3 induced transformation we constructed NIH3T3 cell lines expressing exogenous ShcWT and ShcY317F proteins. The Shc constructs (Figure 1), as well as the pCGN empty vector, produced hygromycin-resistant NIH3T3 colonies with equivalent efficiency (data not shown), thus demonstrating that the overexpression of wild type and Y317F Shc proteins does not interfere with the growth of normal NIH3T3 cells. Several colonies were isolated and analysed for the expression of the transfected Shc constructs (data not shown). Selected cell lines (clone NWT for ShcWT and clone NY317F for ShcY317F) were used as recipients for focus formation assay. Both cell lines were transformed by Ha-ras and TRK-T3 oncogenes with comparable efficiency (data not shown). However, whereas the Ha-ras foci displayed similar size in both recipient cell lines (data not shown), the foci induced by TRKT3 in NY317F cells were smaller than those in NIH3T3 and NWT (Figure 4C). This difference is related to the inhibitory effect of ShcY317F rather than the growth rate of the NY317F parental cell line, as deduced by growth curve determination (unpublished observations).

In order to characterise further the inhibitory effect of ShcY317F on TRK-T3 transforming activity, we investigated the influence of the level of Shc mutant protein expression. To this aim we performed foci selection in the presence of hygromycin, whose resistance is carried by the pCGN expression vector, based on previous observations showing that the level of ShcWT and ShcY317F proteins increases when NWT and NY317F cells are cultured in the presence of the antibiotic (unpublished results). As shown in Figure 4D, the addition of hygromycin did not affect the transforming activity of TRK-T3 in NWT cells. On the contrary, hygromycin reduced the number of foci induced by TRK-T3 in NY317F cells by about $60 \%$, both in the presence and in the absence of G418, whose resistance is carried by the TRK-T3 expression vector. The presence of hygromycin also reduced the size of foci in NY317F cells (data not shown). These results support the view that ShcY317F exerts an inhibitory effect on TRK-T3 transforming activity, which can be modulated by the protein amount.

\section{Long-term effects of ShcY317F on the TRK-T3 induced transformed phenotype}

Having shown that ShcY317F reduced TRK-T3 transforming activity, we were interested in determining any long-term effect in foci 
A
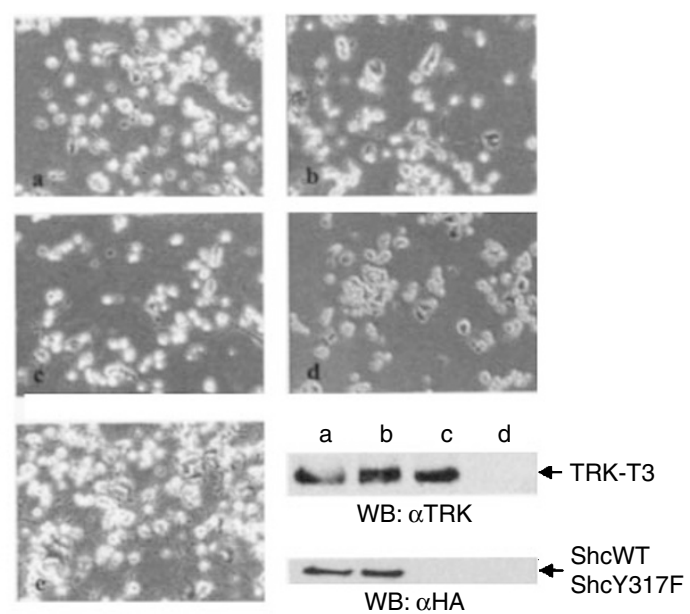

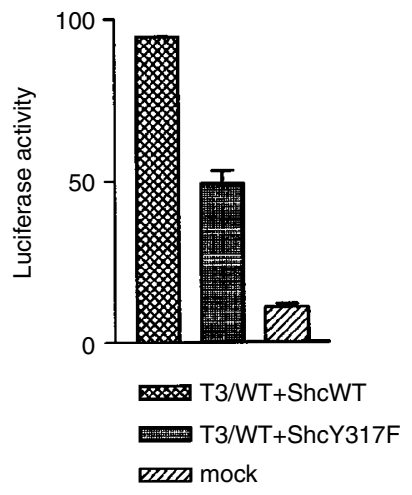

B

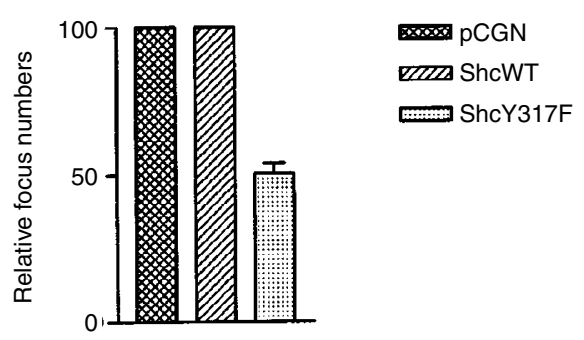

C

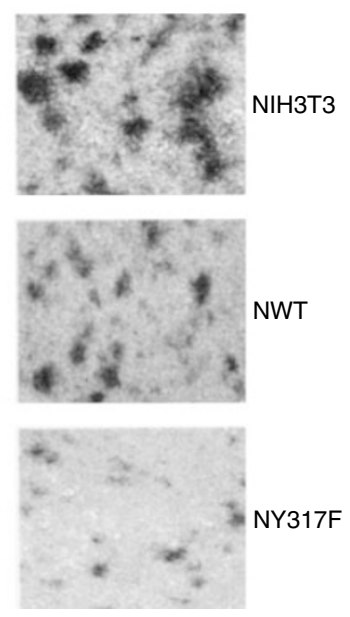

D
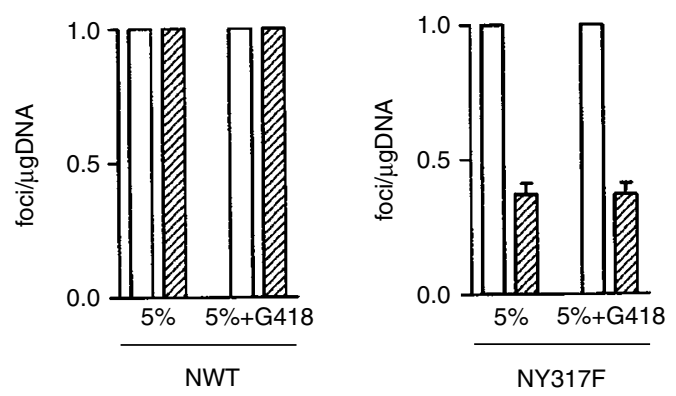

Figure 4 (A) Effect of ShcY3I7F on TRK-T3 induced PCI2 differentiation. PCI 2 cells were cotransfected with TRK-T3 and ShcWT (a), ShcY3 I7F (b) or pCGN (c) plasmids, together with VGF8-luc and Renilla plasmids, as described in Materials and Methods and scored for the presence of neurites 3 days later. As control, untreated (d) and NGF-stimulated $\left(50 \mathrm{ng} \mathrm{ml}^{-1}\right.$ ) (e) PCI 2 cells are shown. After morphological analysis, transfected PCI 2 cells were lysates and subjected to immunoblot using anti-TRK and anti-HA antibodies to detect the level of transfected proteins. To quantify the TRK-T3 differentiating activity both luciferase activities were measured using the Dual-Luciferase reported assay system (Promega). The values were expressed relative to Renilla luciferase activity for normalisation. The results presented are an average of three experiments. (B) Microfocus formation assay. One hundred cells from focus NF797 (NIH3T3 cells transformed by TRK-T3 oncogene) transfected with pCGN vector, ShcWT or ShcY3I7F constructs were combined with I $\times 10^{5} \mathrm{NIH} 3 \mathrm{~T} 3$ cells in 10-cm dishes, and cultured for 2 weeks in medium containing $5 \%$ calf serum. The foci were counted after GIEMSA staining. (C) Transforming activity of TRK-T3 in NIH3T3, NWT (NIH3T3 cells stably expressing ShcWT) and NY3 I7F (NIH3T3 cells stably expressing ShcY3 I7F) cells. Transfection and foci selection were performed as described in Materials and Methods. Foci and G4I 8 resistant colonies were either fixed and GIEMSA stained or isolated for further analysis after two weeks of selection. (D) Effect of hygromycin on TRK-T3 foci formation in NWT and NY3I7F cell lines. Foci selection was performed in $5 \%$ serum medium supplemented or not with $400 \mu \mathrm{g} \mathrm{ml} \mathrm{l}^{-1}$ of G4I8 (selectable marker contained in the TRK-T3 expression vector) in the absence or presence of $25 \mu \mathrm{g} \mathrm{ml}{ }^{-1}$ hygromycin (selectable marker contained in the Shc expression vector). Foci were scored after 2 weeks of selection. For each cell line, the number of foci per $\mu \mathrm{g}$ DNA selected in the absence of hygromycin was set at $100 \%$. 
induced by TRK-T3 oncogene in NY317F cells, expressing the ShcY317F mutant. Several foci were isolated, in the presence or absence of hygromycin and/or G418 (as above reported, Figure $4 \mathrm{D})$ with the aim of enhancing the expression of ShcY317F and/ or TRK-T3 proteins. Interestingly, a difference was evident after isolation: the morphology of the TRK-T3 foci in NY317F cells was less transformed than that of the foci in NIH3T3 and NWT cells, and will be subsequently called 'intermediate' (Table 1). Soon after isolation ('early' in Table 1), Western blot analysis and/or immunofluorescence revealed that all the foci in NY317F cells expressed both TRK-T3 and ShcY317F proteins (data not shown). Later on the behaviour of the foci during culture varied according to the method of selection. The foci selected in the presence of hygromycin, which increased the level of ShcY317F protein, underwent a growth crisis starting 4 weeks after isolation. Most of the cells died; the surviving cells had a flat phenotype, and they could be rescued and propagated by increasing the serum concentration up to $10 \%$ ('late' in Table 1). At this stage Western blot and/or immunofluorescence analysis of the rescued cells showed loss of TRK-T3 expression, but no changes in ShcY317F expression. The same effect was observed in half of the foci isolated in the absence of selective agents, whereas the remaining 50\% maintained the intermediate phenotype and the expression of TRK-T3 protein, but lost the expression of ShcY317F. The foci selected in the presence of both hygromycin and G418 maintained the intermediate phenotype and the expression of both TRK-T3 and ShcY317F proteins. Altogether these results indicate that TRK-T3 full transforming activity requires signaling through tyrosine 317 of Shc.

To get inside the cell death described above, the TRK-T3 induced NY317F foci were monitored twice a week for the occurrence of apoptosis by TUNEL. Although there was considerable variability among the clones, $2-10 \%$ of TUNEL-positive cells was detected in those undergoing a growth crisis and loosing TRK-T3 expression. By contrast, when the parental NY317F cell line or a NIH3T3 focus transformed by TRK-T3 were analysed, no TUNEL-positive cells were scored. Furthermore, the number of TUNEL-positive cells may have been underestimated because TUNEL picks up the late stage of apoptosis marked by DNA breaks. These results suggest that the inhibitory effect of ShcY317F on TRK-T3 transforming activity involves apoptosis.

The effect of selective agents on the transformed phenotype and ShcY317F and TRK-T3 protein expression was recapitulated on focus $3.9 \mathrm{HG}$, which was selected and expanded in the presence of both hygromycin and G418, and had an 'intermediate' phenotype. The cells were cultured in the presence and absence of hygromycin and/or G418 (Figure 5). After one month, the cells kept in the absence of selective agents or in the presence of G418 alone were more transformed than those kept in the presence of both agents, whereas those exposed to hygromycin alone were completely flat (Figure 5A). Western blot analysis demonstrated that the level of TRK-T3 protein was enhanced by G418 and that of ShcY317F protein was enhanced by hygromycin. Furthermore, the expressions of TRK-T3 and ShcY317F were inversely correlated, and the transformed and flat phenotypes respectively correlated with high expression of TRK-T3 and ShcY317F (Figure 5B). These results confirm the considerations deduced from the focus formation assay in NY317F cells, and indicate that TRK-T3 induced transformation is maintained only if endogenous Shc signalling is preserved.

\section{DISCUSSION}

TRK oncogenes, associated with a consistent fraction of human papillary thyroid carcinoma, are generated by somatic rearrangements and display constitutive tyrosine kinase activity (Pierotti et al, 1996). The biological effects of rearranged TRK oncogenes recapitulate that of NGF-stimulated wild type NTRK1 receptor. In fact, similarly to the activated receptor counterpart, TRK oncogenes induce transforma- tion of NIH3T3 mouse fibroblasts and differentiation of rat pheochromocytoma PC12 cells. Analysis of TRK oncogenes signal transduction, performed in NIH3T3 foci, have detected the involvement of several signal transducers recruited by the NGF-stimulated NTRK1 receptor, such as Shc, PLC $\gamma$, ERK1/2 and JNK MAP kinases (Borrello et al, 1994, manuscript in preparation).

With the aim to understand the mechanism leading to thyroid transformation, we explored the role of the Shc adaptor protein in the signal transduction triggered by TRK-T3 oncogene. The mutation of TRK-T3 tyrosine 291, corresponding to the tyrosine 490 of the NTRK1 receptor, abolished the interaction with both Shc and FRS2 adaptor proteins. This led to the abrogation of TRK-T3 induced differentiation and transformation, thus demonstrating that signalling through tyrosine 291 is essential for oncogene activity and it cannot be compensated by possible TRK oncogene-specific signal transduction pathways.

To assess more directly the role of Shc in TRK-T3 activity, we used the ShcY317F mutant, carrying the mutation of the tyrosine 317 which, in addition to tyrosines 239/240, is involved in Grb2 recruitment. The ShcY317F mutant has been shown to exert a dominant-negative effect on the endogenous Shc activity in different contexts. In ErbB2-positive breast cancer cell lines, ShcY317F blocks growth by disrupting cell-cycle progression (Stevenson et al, 1999); in NIH3T3 cells it reduces the RET/PTC2 oncogene transforming activity (Mercalli et al, 2001); in adipocytes it suppresses the IGF-induced proliferation (Boney et al, 2000); moreover, a Shc mutant lacking the $\mathrm{CH} 1$ domain that includes Y317, suppresses NIH3T3 transformation induced by the neu oncogene (Li et al, 1999).

The evidence that the ShcY317F mutant is tyrosyl phosphorylated when coexpressed with TRK-T3 indicates that the oncogene targets residues Tyr239/240 of Shc. However, the capabilty of phosphorylated ShcY317F to interact with Grb2 is reduced with respect to ShcWT. The expression of ShcY317F protein did not affect the capability of TRK-T3 to recruit and activate FRS2. Nevertheless, ShcY317F had an inhibitory effect on TRK-T3 induced differentiation and transformation, thus supporting the hypothesis that Shc signalling through Y317 is indispensable for TRK-T3 activity. Our data on NIH3T3 transformation showed that such inhibitory effect depends on the relative amount of TRK-T3 and ShcY317F proteins. Indeed, inhibition is more evident by adding hygromycin to the selection medium: this increases the amount of ShcY317F protein, thus producing the greatest competition with endogenous Shc for binding to TRK-T3. ShcY317F produced effect not only on the foci number, but also on their morphology, which was manifest upon isolation: foci arose in the presence of ShcY317F were less transformed, and were named 'intermediate'. In addition to these short-term effects on transforming activity, a long-term effect was observed in the cell lines expressing both TRK-T3 and ShcY317F proteins selected as foci of 'intermediate' phenotype. In most cases the clones underwent cell death, and the surviving population lost the transformed morphology and TRK-T3 oncogene expression. Other clones maintained the transformed morphology and oncogene expression either because the ShcY317F protein was not sufficient to titer out the wild type or because they compensated the inhibitory effect of ShcY317F by accessory mechanisms. The inhibition of TRK-T3 activity by ShcY317F is therefore related to effect on both proliferation and transformation.

In clones undergoing cell death we observed a significant number of apoptotic cells. This finding is in keeping with recent data suggesting that Shc plays a role in promoting cell survival and counteracting apoptosis in response to cytokine receptors (Gu et al, 2000) and NGF receptor (Ulrich et al, 1998; Ashcroft et al, 1999) stimulation. The occurrence of apoptosis in our experimental system can be explained by several hypotheses: (1) Shc might transduce possible TRK-T3 anti-apoptotic signals; (2) signalling through tyrosine 317 of Shc could counteract a possible pro- 
Table I Effect of ShcY3I7F on TRK-T3 induced transformed phenotype

\begin{tabular}{|c|c|c|c|c|c|c|c|c|c|}
\hline \multirow[b]{2}{*}{ Selective agent } & \multirow[b]{2}{*}{ Trans. Eff. } & \multicolumn{3}{|c|}{ Early } & \multicolumn{2}{|c|}{$\begin{array}{l}\text { Crisis, apoptosis } \\
\text { and rescue }\end{array}$} & \multicolumn{3}{|c|}{ Late } \\
\hline & & Morph. & $\begin{array}{c}\text { TRK-T3 } \\
\text { expression }\end{array}$ & $\begin{array}{l}\text { ShcY3I7f } \\
\text { expression }\end{array}$ & + & - & Morph. & $\begin{array}{c}\text { TRK } \\
\text { expression }\end{array}$ & $\begin{array}{l}\text { ShcY3I7F } \\
\text { expression }\end{array}$ \\
\hline Hyg & 0.5 & $10 / 101$ & + & + & $10 / 10$ & & $\mathrm{~F}$ & - & + \\
\hline \multirow[t]{2}{*}{ None } & I & $10 / 101$ & + & + & $5 / 10$ & & $\mathrm{~F}$ & - & + \\
\hline & & & & & & $5 / 10$ & i & + & - \\
\hline Hyg+G4I8 & 0.5 & 10/101 & + & + & & $10 / 10$ & I & + & + \\
\hline
\end{tabular}

Early: Weeks $0-3$ after isolation. Late: Weeks 4-6 after isolation. Transf. Eff.: Transforming efficiency. Morph.: Morphology. I: Intermediate. F: Flat. Hyg: Hygromycin. Analysis of morphology, TRK-T3 and ShCY3I7F expression on foci isolated from NY3I7F cells transfected with TRK-T3 and selected in medium without selective agents or containing hygromycin $\left(25 \mu \mathrm{g} \mathrm{ml}{ }^{-1}\right)$ or hygromycin plus $\mathrm{G} 4 \mathrm{l} 8\left(400 \mu \mathrm{g} \mathrm{ml} \mathrm{l}^{-1}\right)$. Ten foci from each selection type were analysed.
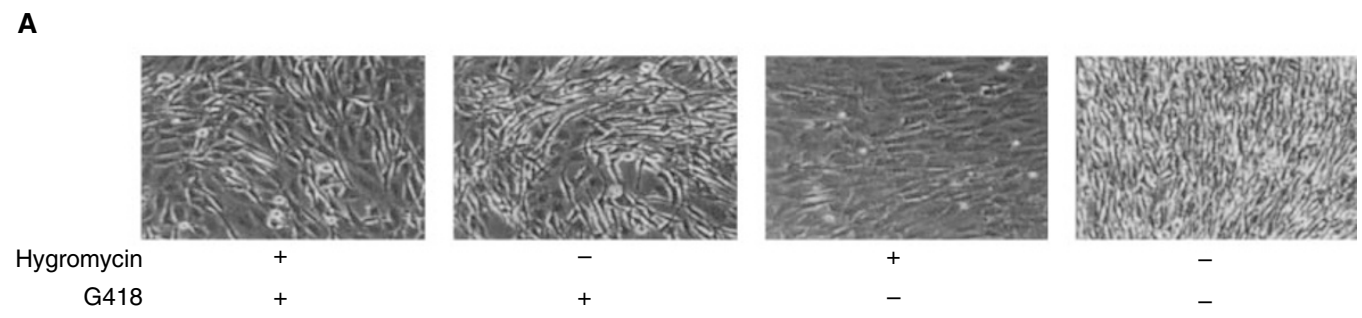

B
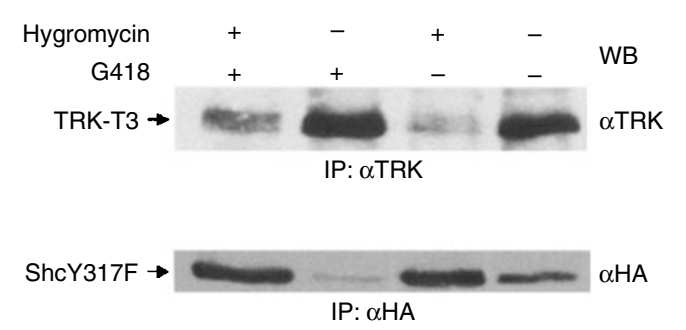

Figure 5 Effect of selective agents on the phenotype and TRK-T3 and ShcY3I7F protein expression on focus 3.9HG. (A) The 3.9HG cell line, generated by transfection of NY3 I 7F cells with TRK-T3, was selected in medium containing both hygromycin $\left(25 \mu \mathrm{g} \mathrm{ml}^{-1}\right)$ and G4l8 (400 $\left.\mu \mathrm{g} \mathrm{ml}{ }^{-1}\right)$ and displayed an 'intermediate' phenotype. The 3.9HG cells were cultured for four weeks in the absence or in the presence of different combinations of selective agents (hygromycin+G418, G418, hygromycin). (B) Cell extracts from the 3.9HG cells cultured under the four different conditions were immunoprecipitated and immunoblotted with anti-TRK and anti-HA antibodies to show the level of TRK-T3 and ShcY3I7F proteins.

apoptotic pathway induced by TRK-T3; (3) a pro-apoptotic signal might be the consequence of cell stress due to incorrectly transduced TRK-T3 signalling. Interestingly, results similar to ours have been obtained by co-expressing ShcY317F and the Ret/PTC2 oncogene (Mercalli et al, 2001), thus indicating the existence of a mechanism shared by other RTK oncogenes.

In conclusion, our data demonstrate that signalling through ShcY317 is essential for TRK-T3 transformation. This residue may cooperate with Tyr239/240 and/or other pathways in such a way that, when mutated, the signalling is not sufficient for specific endpoints; alternatively, Tyr317 may itself trigger specific pathways. Furthermore, the inhibitory effect of ShcY317F may be due to competition not only with endogenous Shc, but also with other adaptors docking the same TRK-T3 site.
The relevance of these studies is the possibility to block the transforming potential of TRK oncogenes by interfering with signalling through the Y317 residue of Shc. However, the mechanism responsible for this inhibitory effect, in particular the apoptotic process, deserves further investigations.

\section{ACKNOWLEDGEMENTS}

This study was supported by the AIRC (Associazione Italiana per la Ricerca sul Cancro). The authors would like to thank MG Borrello for discussion, S Pagliardini for technical assistance and Cristina Mazzadi for her secretarial assistance. 


\section{REFERENCES}

Ashcroft M, Stephens RM, Hallberg B, Downward J, Kaplan DR (1999) The selective and inducible activation of endogenous PI 3-kinase in PC12 cells results in efficient NGF-mediated survival but defective neurite outgrowth. Oncogene 18: 4586-4597

Boney CM, Gruppuso PA, Faris RA, Frackelton Jr AR (2000) The critical role of Shc in insulin-like growth factor-I-mediated mitogenesis and differentiation in 3T3-L1 preadispocytes. Mol Endocrinol 14: $805-813$

Bongarzone I, Pierotti MA, Monzini N, Mondellini P, Manenti G, Donghi R, Pilotti S, Grieco M, Santoro M, Fusco A, Vecchio G, Della Porta G (1989) High frequency of activation of tyrosine kinase oncogenes in human papillary thyroid carcinoma. Oncogene 4: 1457-1462

Borrello MG, Pelicci G, Arighi E, De Filippis L, Greco A, Bongarzone I, Rizzetti MG, Pelicci PG, Pierotti MA (1994) The oncogenic versions of the Ret and Trk tyrosine kinases bind Shc and Grb2 adaptor proteins. Oncogene 9: $1661-1668$

Canu N, Possenti R, Rinaldi AM, Trani E, Levi A (1997) Molecular cloning and characterization of the human VGF promoter region. J Neurochem 68: $1390-1399$

Dikic I, Batzer AG, Blaikie P, Obermeier A, Ullrich A, Schlessinger J, Margolis B (1995) Shc binding to Nerve Growth Factor receptor is mediated by the phosphotyrosine interaction domain. J Biol Chem 270: 15125-15129

Fournier E, Blaikie P, Rosnet O, Margolis B, Birnbaum D, Borg JP (1999) Role of tyrosine residues and protein interaction domains of SHC adaptor in VEGF receptor 3 signaling. Oncogene 18: $507-514$

Gotoh N, Tojo A, Shibuya M (1996) A novel pathway from phosphorylation of tyrosine residues $239 / 240$ of Shc, contributing to suppress apoptosis by IL-3. EMBO J 15: 6197-6204

Gotoh N, Toyoda M, Shibuya M (1997) Tyrosine phosphorylation sites at amino acids 239 and 240 of Shc are involved in epidermal growth factor-induced mitogenic signaling that is distinct from Ras/mitogen-activated protein kinase activation. Mol Cell Biol 17: 1824-1831

Greco A, Fusetti L, Miranda C, Villa R, Zanotti S, Pagliardini S, Pierotti MA (1998) Role of the TFG N-terminus and coiled-coil domain in the transforming activity of the thyroid TRK-T3 oncogene. Oncogene 16: 809-816

Greco A, Mariani C, Miranda C, Lupas A, Pagliardini S, Pomati M, Pierotti MA (1995) The DNA rearrangement that generates the TRK-T3 oncogene involves a novel gene on chromosome 3 whose product has a potential coiled-coil domain. Mol Cell Biol 15: 6118-6127

Gu H, Maeda H, Moon JJ, Lord JD, Yoakim M, Nelson BH, Neel BG (2000) New role for shc activation of the phosphatidynositol 3-kinase/akt pathway. Mol Cell Biol 20: 7109-7120

Kaplan DR, Miller FD (1997) Signal transduction by the neurotrophin receptors. Curr Opin Cell Biol 9: 213-221

Kouhara H, Hadari YR, Spivak-Kroizman T, Schilling J, Bar-Sagi D, Lax I, Schlessinger J (1997) A lipid-anchored Grb2-binding protein that links FGF-receptor activation to the Ras/MAPK signaling pathway. Cell 89: $693-702$

Li K, Shao R, Hung MC (1999) Collagen-homology domain 1 deletion mutant of Shc suppresses transformation mediated by neu through a MAPK-independent pathway. Oncogene 18: 2617-2626

MacDonald JIS, Gryz EA, Kubu CJ, Verdi JM, Meakin SO (2000) Direct binding of the signaling adapter protein grb2 to the activation loop tyrosines on the nerve growth factor receptor tyrosine kinase, trkA. J Biol Chem 275: $18225-18235$

Meakin SO, MacDonald JIS, Gryz EA, Kubu CJ, Verdi JM (1999) The signaling adapter FRS-2 competes with Shc for binding to the nerve growth factor receptor TrkA. A model for discriminating proliferation and differentiation. J Biol Chem 274: $9861-9870$

Mencinger M, Aman P (1999) Characterization of TFG in mus musculus and Caenorhabditis elegans. Biochem Biophys Res Commun 257: 67-73

Mencinger M, Panagopoulos I, Andreasson P, Lassen C, Mitelman F, Aman P (1997) Characterization and chromosomal mapping of the human TFG gene involved in thyroid carcinoma. Genomics 41: $372-331$
Mercalli E, Ghizzoni S, Arighi E, Alberti L, Sangregorio R, Radice MT, Gishizky ML, Pierotti MA, Borrello MG (2001) Key role of Shc signaling in the transforming pathway triggered by Ret/ptc2 oncoprotein. Oncogene 20: $3475-3485$

Migliaccio E, Giorgio M, Mele S, Pelicci G, Reboldi P, Pandolfi PP, Lanfrancone L, Pelicci PG (1999) The p66shc adaptor protein controls oxidative stess response and life span in mammals. Nature 402: 309-313

Migliaccio E, Mele S, Salcini AE, Pelicci G, Lai KM, Superti-Furga G, Pawson T, Di Fiore PP, Lanfrancone L, Pelicci PG (1997) Opposite effects of the p52shc/p46shc and p66shc splicing isoforms on the EGF receptor-MAP kinase-fos signalling pathway. EMBO J 16: $706-716$

Miranda C, Greco A, Miele C, Pierotti MA, Van Obberghen E (2001) IRS-1 and IRS-2 are recruited by TrkA receptor and oncogenic TRK-T1. J Cell Physiol 186: $35-46$

Muragaki Y, Chou TT, Kaplan DR, Trojanowski JQ, Lee VM (1997) Nerve growth factor induces apoptosis in human medulloblastoma cell lines that express TrkA receptors. J Neurosci 17: 530-542

Obermeier A, Lammers R, Wiesmuller KH, Jung G, Schlessinger J, Ullrich A (1993) Identification of Trk binding sites for SHC and phosphatidylinositol 3'-kinase and formation of a multimeric signaling complex. J Biol Chem 268: $22963-22966$

Ong SH, Guy GR, Hadari YR, Laks S, Gotoh N, Schlessinger J, Lax I (2000) FRS2 proteins recruit intracellular signaling pathways by binding to diverse targets on fibroblast growth factor and nerve growth factor receptors. Mol Cell Biol 20: $979-989$

Pelicci G, Lanfrancone L, Grignani F, McGlade J, Cavallo F, Forni G, Nicoletti I, Pawson T, Pelicci PG (1992) A novel transforming protein (SHC) with an $\mathrm{SH} 2$ domain is implicated in mitogenic signal transduction. Cell 70: $93-104$

Pierotti MA, Bongarzone I, Borrello MG, Greco A, Pilotti S, Sozzi G (1996) Cytogenetics and molecular genetics of the carcinomas arising from the thyroid epithelial follicular cells. Genes Chrom Cancer 16: 1-14

Possenti R, Di Rocco G, Nasi S, Levi A (1992) Regulatory elements in the promoter region of vgf, a nerve growth factor-inducible gene. Proc Natl Acad Sci USA 89: 3815-3819

Qian X, Riccio A, Zhang Y, Ginty DD (1998) Identification and characterization of novel substrates of Trk receptors in developing neurons. Neuron 21: $1017-1029$

Rabin SJ, Cleghon V, Kaplan DR (1993) SNT, a differentiation-specific target of neurotrophic factor-induced tyrosine kinase activity in neurons and PC12 cells. Mol Cell Biol 13: 2203-2213

Stephens RM, Loeb DM, Copeland TD, Pawson T, Greene LA, Kaplan DR (1994) Trk receptors use redundant signal transduction pathways involving SHC and PLC-gammal to mediate NGF responses. Neuron 12: $691-705$

Stevenson LE, Ravichandran KS, Frackelton AR Jr (1999) Shc dominant negative disrupts cell cycle progression in both G0-G1 and G2-M of ErbB2positive breast cancer cells. Cell Growth Diff 10: $61-71$

Thomas D, Bradshaw RA (1997) Differential utilization of ShcA tyrosine residues and functional domains in the transduction of epidermal growth factor-induced mitogen-activated protein kinase activation in 293T cells and nerve growth factor-induced neurite outgrowth in PC12 cells. Identification of a new Grb2.Sos1 binding site. J Biol Chem 272: 22293 - 22299

Ulrich E, Duwel A, Kauffmann-Zeh A, Gilbert C, Lyon D, Rudkin B, Evan G, Martin-Zanca D (1998) Specific TrkA survival signals interfere with different apoptotic pathways. Oncogene 16: 825-832

Van der Geer P, Wiley S, Lai VK, Olivier JP, Gish GD, Stephens R, Kaplan D, Shoelson S, Pawson T (1995) A conserved amino-terminal Shc domain binds to phosphotyrosine motifs in activated receptors and phosphopeptides. Curr Biol 5: 404-412 